\title{
Experimental Study on Age Estimation of Bloodstains Based on Biological and Toxicological Analysis
}

\author{
Ken-Ichiro Nakao ${ }^{*}$, Ryo Shimada $^{1}$, Kenji Hara ${ }^{2}$ and Kazuhiko Kibayashi ${ }^{1}$ \\ ${ }^{I}$ Department of Legal Medicine, School of Medicine, Tokyo Women's Medical University, Tokyo, Japan \\ ${ }^{2}$ Department of Forensic Medicine, Faculty of Medicine, Fukuoka University, Fukuoka, Japan
}

\begin{abstract}
The determination of the age of bloodstains in forensic investigations helps in estimating the time elapsed between incidents (such as accidents or violent crimes wherein the blood was shed) and sampling. In this study, bloodstains containing either ethanol or amphetamine sulfate and methamphetamine hydrochloride were prepared and incubated at $25^{\circ} \mathrm{C}$ and $50 \%$ humidity to determine their age, based on temporal changes in the relative levels of microRNA 16 (miR-16) and miR-451 after incubation. The miR-16 and miR-451 levels decreased significantly 5-28 days after incubation in the chamber. The concentration of ethanol in the bloodstains decreased rapidly during storage and became undetectable after $180 \mathrm{~min}$. The concentrations of amphetamine sulfate and methamphetamine hydrochloride in the bloodstains remained almost constant throughout the experimental period. These findings indicate that the age of bloodstains can be calculated simply on the basis of the relative levels of miRNAs. Although it is difficult to utilize the concentrations of ethanol, methamphetamine, and amphetamine sulfate for estimating the age of the bloodstain, these data could provide evidence of the victim or assailant having used these substances at the scene of an accident or crime.
\end{abstract}

Keywords: Bloodstain, ethanol, amphetamine, methamphetamine, microRNA.

\section{INTRODUCTION}

Bloodstain samples from crime scenes are important evidence in forensic investigations [1]. Accurate determination of the age of bloodstains provides a temporal connection between the bloodstains and incidents such as murder and physical injury as well as the time elapsed between the incident and the collection of bloodstain samples [2]. Recent studies have shown that RNA degradation rates are useful for estimating the age of biological specimens [3]. MicroRNAs (miRNAs) belong to the class of endogenous, small, 18-24-nucleotide long, noncoding RNA molecules that regulate gene expression at the post-transcriptional level. Hanson et al. [4] reported that the blood-specific miRNAs miR-16 and miR-451are stable in dried bloodstains.

The detection of alcohol or drugs in bloodstains is essential to determine whether the victim or the assailant injured at the scene was under the influence of either. It may also be possible to estimate the age of bloodstains and therefore the time elapsed since the incident based on the concentration of alcohol or drugs in the blood of the injured persons at the time of injury. However, only few studies have estimated the concentrations of alcohol and drugs in blood at the time of injury by determining their concentrations in bloodstains sampled from the crime scene. Alcohol or drug addicts are frequently involved in assaults

*Address correspondence to this author at the Department of Legal Medicine, Tokyo Women's Medical University School of Medicine, 8-1 Kawada-cho, Shinjuku-ku, Tokyo 162-8666, Japan;

Tel/Fax: +81-3-5269-7300; E-mail: knakao55@ research.twmu.ac.jp because of their lifestyle; when they are injured, they often leave bloodstains at the scene of injury. In addition, the use of alcohol and illegal drugs increases the risk of severe and fatal accidents [5, 6]. Therefore, in medico-legal investigations, the concentrations of alcohol and drugs in bloodstains are required to estimate the concentrations of these substances in the blood of the injured individual at the time of an incident. This helps the investigators understand the psychological state of the victim or assailant at the crime scene and, thereby, helps determine the cause of the incident.

In this study, we aimed to determine the age of bloodstains using real-time quantitative polymerase chain reaction (PCR) analyses for miR-16 and miR-451. In addition, we studied the temporal changes in the concentrations of ethanol, amphetamine sulfate, and methamphetamine hydrochloride in the sampled bloodstains to calculate their age. Because methamphetamine and its metabolite amphetamine are widely abused and frequently encountered in medicolegal investigations, we selected these drugs for this study [7, 8].

\section{MATERIALS AND METHODOLOGY}

\section{Reagents}

Human whole blood containing heparin sodium was purchased from Biopredic International (Rennes, France) and stored at $-20^{\circ} \mathrm{C}$ until use. Methamphetamine hydrochloride was purchased from Dainippon Pharma (Osaka, Japan). Amphetamine sulfate was synthesized by one of the authors (K.H) with the permission of the Ministry of Health, Labor, and Welfare. Ethanol, tert-butanol, methanol, acetonitrile, $0.1 \%$ trifluoroacetic acid-acetonitrile, $0.2 \%$ ammonia- 
acetonitrile, sodium acetate, acetic anhydride, and ethyl acetate were purchased from Wako Pure Chemical Industries (Osaka, Japan). Diazepam-D5 in ethyl acetate and pyridine were purchased from Hayashi Pure Chemical Industries (Osaka, Japan) and Thermo Scientific (Milwaukee, WI, USA), respectively. All chemicals used in the present study were of analytical reagent grade.

\section{Bloodstains}

A total of 115 bloodstain samples were analyzed in this study. For the miRNA analysis, $1 \mathrm{~mL}$ blood was placed on a piece of white unbleached cotton $(2 \times 2 \times 1 \mathrm{~cm}, 160-190$ $\mathrm{mg}$ ); the soaked cotton piece was then placed on plastic Petri dishes and left in a chamber (HPAV-48-20; Isuzu Seisakusho, Niigata, Japan) regulated at $25^{\circ} \mathrm{C}$ and $50 \%$ humidity for $0,1,3,5,7,14,21$, or 28 days $(n=5$ per time point, total $=40)$. For gas chromatography $(\mathrm{GC})$ analysis, 1 $\mathrm{mL}$ blood containing $2 \mathrm{mg} / \mathrm{mL}$ ethanol was placed on a similar piece of white unbleached cotton. The cotton piece was placed on plastic Petri dishes and then left in the chamber for $0,30,60,90,120,150$, or $180 \mathrm{~min}(n=5$ per time point, total $=35$ ). For GC-mass spectrometry (GC-MS) analysis, $1 \mathrm{~mL}$ blood containing $0.1 \mu \mathrm{g} / \mathrm{mL}$ amphetamine sulfate and $1 \mu \mathrm{g} / \mathrm{mL}$ methamphetamine hydrochloride was placed on a similar piece of white unbleached cotton. The cotton piece was subsequently placed on plastic Petri dishes and left in the chamber for $0,1,3,5,7,14,21$, or 28 days ( $n$ $=5$ per time point, total $=40$ ). At the end of the incubation period, the bloodstain samples were cut into 4 strips and placed in a $15-\mathrm{mL}$ BD Falcon ${ }^{\mathrm{TM}}$ tube or a $20-\mathrm{mL}$ headspace vial.

\section{Isolation of Leukocytes from Dried Bloodstains}

The dried bloodstains on Petri dishes were collected into 50-mL BD Falcon ${ }^{\mathrm{TM}}$ tubes; $5 \mathrm{~mL}$ Buffer EL (Qiagen, Hilden, Germany) was directly added and the tubes were vortexed for $30 \mathrm{~min}$ at $4{ }^{\circ} \mathrm{C}$. The mixtures were transferred to fresh $15-\mathrm{mL}$ tubes using plastic syringes. The tubes were incubated for $20 \mathrm{~min}$ on ice and briefly vortexed twice during the incubation period. The samples were then centrifuged for $10 \mathrm{~min}$ at $4^{\circ} \mathrm{C}$. The supernatants were removed completely and added to $2 \mathrm{~mL}$ Buffer EL, followed by brief vortexing. The samples were centrifuged for $10 \mathrm{~min}$ at $4^{\circ} \mathrm{C}$, and the supernatants were removed and discarded. Thus, leukocyte pellets were obtained. All centrifugations were performed at $800 \times g$ and $4{ }^{\circ} \mathrm{C}$.

\section{Total RNA Extraction and Purification}

A total of $700 \mu \mathrm{L}$ QIAzol Lysis Reagent (Qiagen) was added to the leukocyte pellet in a tube and mixed with a pipette. The mixture was then added to the tube containing the homogenate and kept on a bench-top for $5 \mathrm{~min}$ at room temperature. Chloroform $(140 \mu \mathrm{L})$ was subsequently added to this tube. The cap was secured, and the tube was shaken vigorously for $15 \mathrm{~s}$. The tube was allowed to stand for $3 \mathrm{~min}$ at room temperature and then centrifuged for $15 \mathrm{~min}$ at $12,000 \times g$ and $4{ }^{\circ} \mathrm{C}$. The upper aqueous phase was transferred to a new reaction tube, and $350 \mu \mathrm{L}$ of $70 \%$ ethanol was added. The samples were transferred to an
RNeasy Mini Spin Column (Qiagen), which was placed in a fresh 2-mL collection tube. The lid of this tube was closed gently, and the tube was centrifuged at $10,000 \times g$ for $15 \mathrm{~s}$ at room temperature. The flow-through, which contained the miRNA, was collected in a fresh collection tube. The miRNA-enriched fraction from the flow-through was purified with the RNeasy MiniElute Cleanup Kit (Qiagen) according to the manufacturer's instructions. Finally, the miRNA-enriched fraction was eluted with $14 \mu \mathrm{L}$ RNase-free water.

\section{Complementary DNA Synthesis and Real-Time PCR}

The cDNA for the real-time PCR was created using the miScript II RT Kit (Qiagen) according to the manufacturer's instructions. The reverse transcriptase reactions were performed in a final volume of $20 \mu \mathrm{L}$ in the GeneAmp ${ }^{\circledR}$ PCR System 9700 . The reaction mixtures were incubated at $37^{\circ} \mathrm{C}$ for $60 \mathrm{~min}$ and then at $95^{\circ} \mathrm{C}$ for $5 \mathrm{~min}$ to inactivate the reverse transcriptase.

Real-time PCRs were performed in Applied Bio-systems 7500 using the miScript SYBR Green PCR Kit (Qiagen). HsRNU6-2 (U6b) miScript Primer Assay, Hs-miR-16-1 (miR16) miScript Primer Assay, and Hs-miR-451-1 (miR-451) miScript Primer Assay were purchased from Qiagen (Hilden, Germany). U6b was used as a normalization standard to correct for intragroup and intergroup variations in between the samples and runs [4]. The cycling parameters were as follows: activation for $15 \mathrm{~min}$ at $95^{\circ} \mathrm{C}$ and 45 cycles of denaturation for $15 \mathrm{~s}$ at $94^{\circ} \mathrm{C}$, annealing for $30 \mathrm{~s}$ at $55^{\circ} \mathrm{C}$, and extension for $34 \mathrm{~s}$ at $70^{\circ} \mathrm{C}$. The expression levels were normalized to that of U6b. Electrophoresis and melting curve analysis were performed after real-time PCR to confirm that the constructed primer pair did not produce any by-products. Control amplifications were performed without cDNA. Relative quantifications of both miR-16 and miR-451 concentrations were performed using the $2^{-\Delta \Delta \mathrm{Ct}}$ method.

\section{GC and GC-MS Analyses}

In the GC analysis for ethanol, distilled water of the same mass as that of the cotton and $200 \mu \mathrm{L} 0.01 \%$ tert-butanol as an internal standard were added to a $20-\mathrm{mL}$ headspace vial containing a bloodstain sample. The vial was sealed with silicone/polytetrafluoroethylene septa and aluminum caps and heated for $15 \mathrm{~min}$ at $55^{\circ} \mathrm{C}$ in the vial rack of an HSS-2B automatic sampler (Shimadzu, Kyoto, Japan). The ethanol concentrations in the cotton pieces were determined using a Shimadzu GC-14A gas chromatograph with a glass column $(2.1 \mathrm{~m} \times 3.2 \mathrm{~mm}$ internal diameter [i.d.], 10\% PEG 600 stationary phase) and a flame ionization detector. The column temperature was set at $90^{\circ} \mathrm{C}$. The temperatures of the injector and detector were both set at $140^{\circ} \mathrm{C}$. Nitrogen was used as a carrier gas under a pressure of $0.5 \mathrm{~kg} / \mathrm{cm}^{3}$. The cutoff value was set at $0.1 \mathrm{mg} / \mathrm{mL}$.

In the GC-MS analyses for methamphetamine and amphetamine, cotton pieces were homogenized in a $15-\mathrm{mL}$ tube using a mixture containing $4 \mathrm{~mL}$ distilled water, $300 \mu \mathrm{L}$ $1 \mathrm{M}$ acetic acid-sodium acetate, and $100 \mu \mathrm{L}$ internal standard solution (IS,10 ppm diazepam-d5 in ethyl acetate). The samples were shaken for $5 \mathrm{~min}$ and centrifuged at 3,000 rpm for $20 \mathrm{~min}$. The upper layer was transferred to a solid-phase 
extraction (SPE) column conditioned with methanol and distilled water. The column was then rinsed with $10 \%$ acetonitrile and eluted with $0.1 \%$ trifluoroacetic acidacetonitrile and $0.2 \%$ ammonia-acetonitrile. The eluted sample was kept at $60^{\circ} \mathrm{C}$ and dried under a nitrogen stream. The residue was dissolved in $50 \mu \mathrm{L}$ acetic anhydride, and $50 \mu \mathrm{L}$ pyridine was added for acetylation. The mixture was kept at $60^{\circ} \mathrm{C}$ for $30 \mathrm{~min}$ and dried under a nitrogen stream at room temperature. Finally, the residue was dissolved in 100 $\mu \mathrm{L}$ ethyl acetate.

GC-MS analysis was performed on an Agilent 6850 gas chromatograph interfaced with an Agilent 5975C mass-selective detector. An HP-5MS column (5\% phenyl methyl siloxane, 30 $\mathrm{m} \times 0.25 \mathrm{~mm}$ i.d., $0.25 \mu \mathrm{m}$ film thickness) was used with helium as the carrier gas at a flow rate of $1.0 \mathrm{~mL} / \mathrm{min}(6850$ Series Auto Sampler). The splitless injection mode was selected with a valve off-time of $2 \mathrm{~min}$, and $2 \mu \mathrm{L}$ of the sample was injected. The electron impact ionization energy was $70 \mathrm{eV}$. The operating conditions for the analyses were as follows: injection port, $250^{\circ} \mathrm{C}$; detector, $280^{\circ} \mathrm{C}$; initial oven temperature, $60^{\circ} \mathrm{C}$ for $2 \mathrm{~min}$, increasing at a rate of $20^{\circ} \mathrm{C} / \mathrm{min}$ to $300^{\circ} \mathrm{C}$, and maintained at $300^{\circ} \mathrm{C}$ for $10 \mathrm{~min}$. The MS was operated in the selected-ion monitoring (SIM) mode; the ions selected for monitoring were chosen from full-scan mass spectral analyses of the analysis that gave minimum interference. The following ions were used as targets and 2 qualifier ions: $m / z 86$ and 91 , 118 for amphetamine; $\mathrm{m} / \mathrm{z} 58$ and 100,117 for methamphetamine and $\mathrm{m} / \mathrm{z}, 261$ and 287, 289 for the IS (diazepam-d5), respectively. SPE was performed using the GLSPE Vacuum Manifold Kit and FOCUS ${ }^{\text {TM }}$ Polar-Enhanced SPE Columns with polar-enhanced styrene-divinylbenzene polymer.

\section{Statistics}

All values were expressed as mean \pm standard deviation (SD). Differences between the values at day 0 and at other time points were determined by one-way analysis of variance (ANOVA), followed by Tukey's post hoc test. The level of significance was set as $p<0.05$.

\section{RESULTS}

\section{miRNA Concentrations in Bloodstains}

After incubation in the chamber, the relative levels of miR-16 and miR-451 showed a tendency to decrease with time (Fig. 1A). The relative levels of miR-16 on days 5, 7, and 21 after incubation were significantly lower than that on day $0(p<0.05)$. The relative levels of miR-451, after 7-28 days of incubation, were significantly lower than that on day $0(p<0.05)$. Fig. (1B) shows the log transformations of the relative levels of miR-16 and miR-451. The linear equations of the miR-16 and miR-451 concentrations were as follows: $\mathrm{y}=(-0.0126) \times(-0.1636)$ and $\mathrm{y}=(-0.0361) \times(-0.1002)$, respectively. The correlation coefficients of the miR-16 and miR-451 concentrations were 0.409 and 0.871 , respectively.

\section{Ethanol Concentrations in Bloodstains}

GC analysis showed that the concentration of ethanol in the bloodstains after incubation decreased significantly over

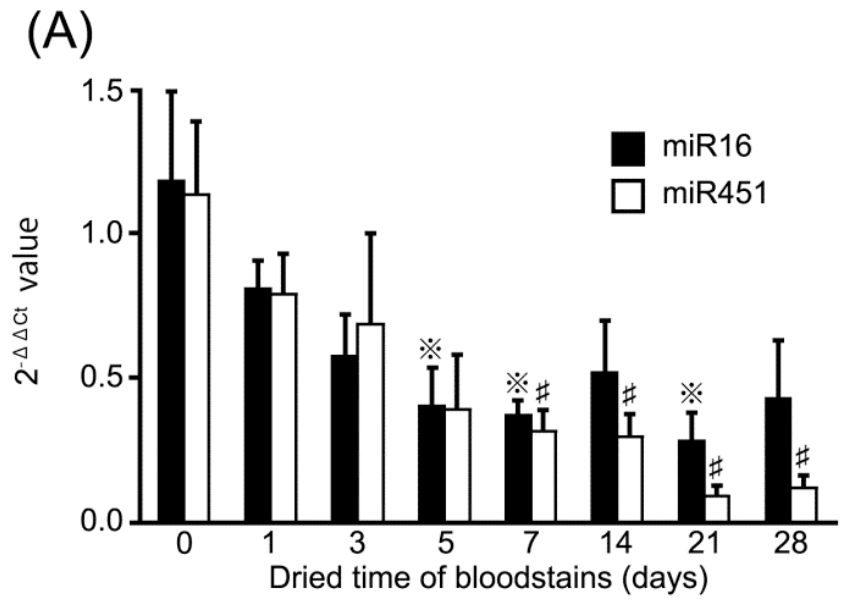

(B)

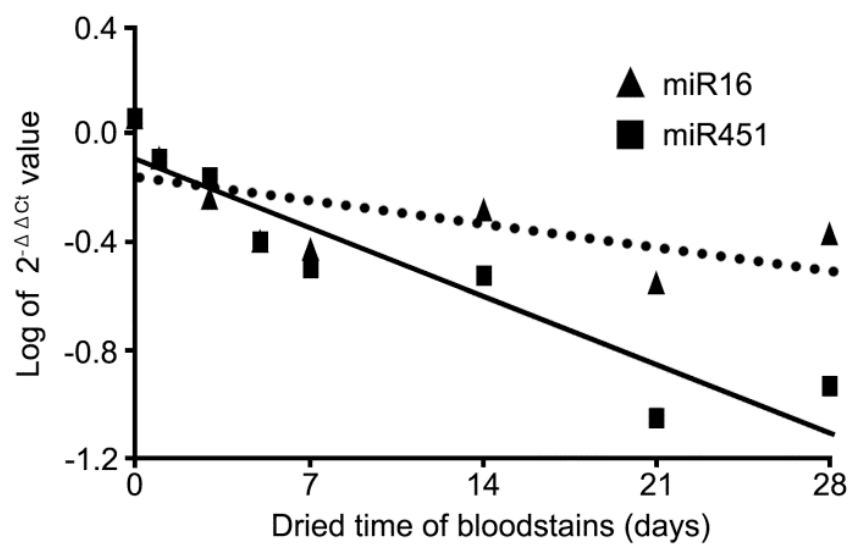

Fig. (1). Relative quantification microRNA 16 (miR-16) and miR451 levels, 0-28 days after introduction into the incubation chamber (A). Values are expressed as mean \pm standard deviation $(n=5$ at each time point; ${ }^{*} p<0.05$ compared with the level of miR-16 at day $0 ;{ }^{\#}<0.05$ compared with the level of miR-451 at day 0 . (B) Log of the $2^{-\Delta \Delta \mathrm{Ct}}$ values. The dotted line represents the linear equation of miR-16 and the straight line represents the linear equation of miR451.

time compared to that prior to incubation ( $0 \mathrm{~min} ; p<0.05)$. There was a rapid decrease in the ethanol concentration in the bloodstains during the first $30 \mathrm{~min}$ after removal from the chamber; the ethanol concentrations approached the cutoff value at $150 \mathrm{~min}$ and were undetectable at $180 \mathrm{~min}$ (Fig. 2A). The log transformation of the ethanol concentrations yielded the following linear equation: $y=(-0.0184) \times$ $(+0.3076)$ with a correlation coefficient of 0.966 (Fig. 2B).

\section{Amphetamine and Methamphetamine Concentrations in Bloodstains}

GC-MS analysis revealed that amphetamine sulfate concentrations in the bloodstains were significantly higher at 3 days after incubation in the chamber than on day $0(p<$ 0.05 ) (Fig. 3A). However, there was no significant difference in the methamphetamine hydrochloride concentration throughout the experimental period relative to the concentration of day 0 (Fig. 3B). Approximations of each 

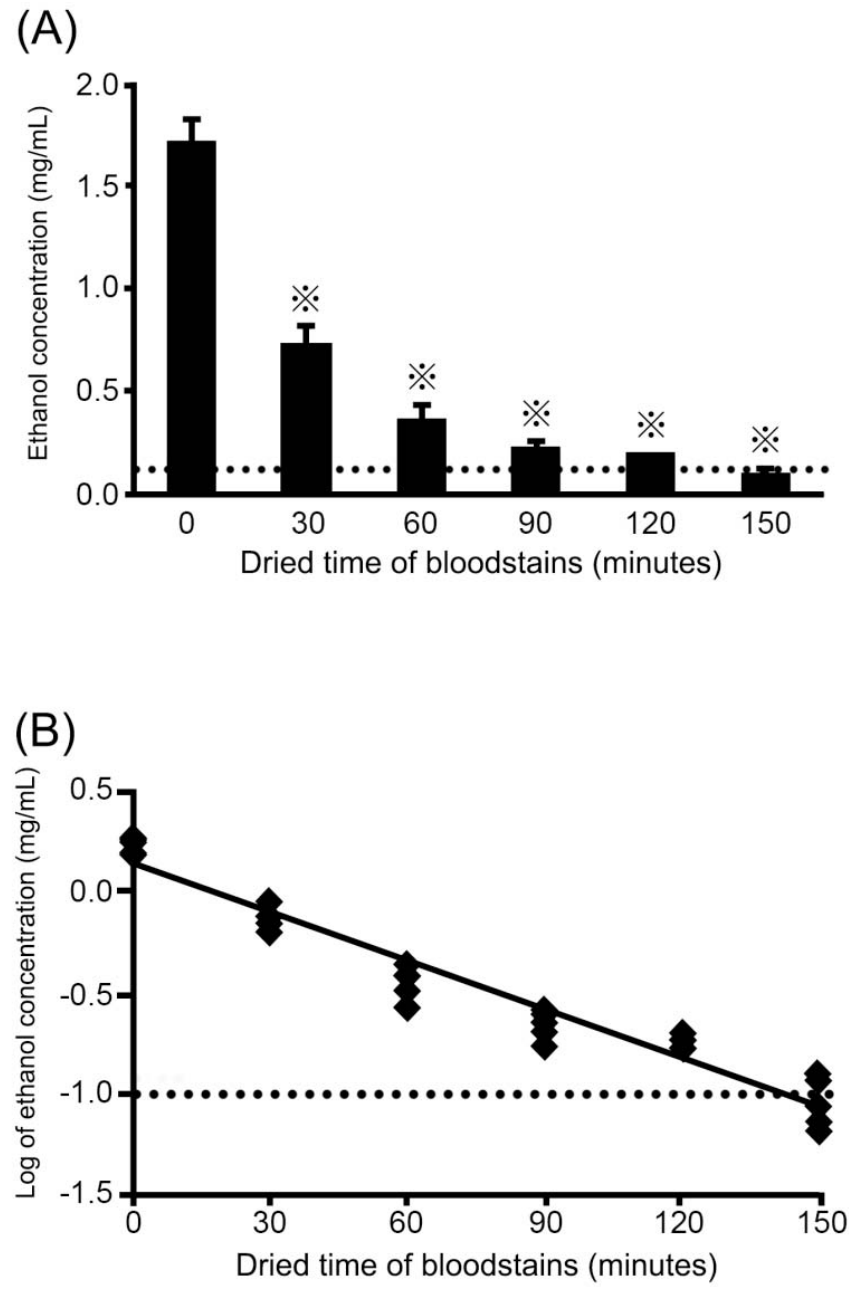

Fig. (2). (A) Ethanol concentration in dried bloodstains as assessed using gas chromatography at $0-180 \mathrm{~min}$ after introduction into the incubation chamber. Values are expressed as mean \pm standard deviation ( $\mathrm{n}=5$ at each time point; ${ }^{*} p<0.05$ compared with the ethanol concentration at $0 \mathrm{~min}$ ). The data point for $180 \mathrm{~min}$ is not shown because this value was below the cutoff value (dotted line). (B) Ethanol concentration log. The dotted line indicates the cutoff value.

concentration during the experimental period showed that the initial concentrations were accurately approximated (Figs. 3C , D). The linear equations of amphetamine sulfate and methamphetamine hydrochloride concentrations were as follows: $\mathrm{y}=(-0.046) \times(+0.9697)$ and $\mathrm{y}=(-0.0004) \times$ $(+0.1006)$, respectively.

\section{DISCUSSION}

Bloodstain samples are among the most important pieces of forensic evidence at crime and accident scenes. Estimating the age of bloodstains at crime and accident scenes provides a temporal connection between the bloodstains and the incident. In addition, if ethanol, illegal drugs, or medications can be detected in a bloodstain, the concentrations of these substances in the blood at the time of bloodstain formation can be calculated. In this study, we investigated the relative levels of blood-specific miRNAs to determine the age of bloodstains and to assess the temporal changes in the ethanol, amphetamine sulfate, and methamphetamine hydrochloride concentrations in bloodstain samples incubated at $25^{\circ} \mathrm{C}$ and $50 \%$ humidity. The relative levels of miR-16 after 5, 7, and 21 days of incubation in the chamber were significantly lower than on day 0 . Furthermore, the levels of miR-451 were significantly lower after 7-28 days of incubation than on day 0 . These findings indicate biological degradation of the bloodstains. The relative levels of miR-16 on days 14 and 28 were not significantly lower than on day 0 ; this result is probably because the miR-16 concentrations measured on these days were outliers. Logarithmic conversion of the relative levels of miR-16 and miR-451 yielded linear equations. These findings indicate that analyzing miR-16 and miR-451 levels could be useful for estimating the age of a bloodstain. In contrast, ethanol concentrations in the bloodstains decreased rapidly between 30 and $150 \mathrm{~min}$ after incubation and became undetectable thereafter. The concentrations of amphetamine sulfate and methamphetamine hydrochloride in the bloodstain samples remained constant for 28 days; however, the concentration of amphetamine sulfate measured at day 3 was significantly elevated, which was due to variability during the extraction process. These findings indicate that it is difficult to estimate the age of a bloodstain on the basis of the concentrations of ethanol, amphetamine sulfate, and methamphetamine hydrochloride. However, the results of this study indicate that it is possible to detect these substances in the bloodstains as evidence that the victim or assailant consumed these substances at the time of injury or at the crime scene.

In a previous study, researchers determined the age of bloodstains using biological elements such as miRNAs [4]. Although miRNAs are among the most stable elements in blood, they tend to degrade when bloodstains dry incompletely and under high humidity $[9,10]$. Bremmeret al. [2] recommended humidity levels of less than $40 \%$ for experiments on bloodstain ageing. However, in the present study, we placed bloodstains in a chamber at $25^{\circ} \mathrm{C}$ and $50 \%$ humidity. The significant decrease in the miR-16 and miR451 concentrations in the bloodstains can be explained by the fact that the bloodstains were not dried before being placed in the chamber; moreover, the humidity level in the chamber was $50 \%$. Wet bloodstains are more suitable for use in medico-legal investigations than dried ones. The results show that reductions in the relative levels of miR-16 and miR-451 may be useful for estimating the age of bloodstains. Because U6b was used as a normalization standard, comparison with fresh blood was not necessary for miRNA analyses of bloodstains.

Alcohol consumption is one of the main factors responsible for fatal motor vehicle crashes. Drunk driving generally plays a major role in traffic accidents, especially in severe and fatal accidents $[11,12]$. Therefore, blood alcohol concentrations are measured in injured drivers who have been transferred to hospitals. However, the alcohol concentration may not be measured in all patients immediately after the accident. Identifying alcohol in bloodstains on the road surface and vehicle interiors may help identify drunk drivers when investigating motor vehicle accidents. Xiaoqin et al. [13] examined blood alcohol concentrations in previously opened and unopened tubes. The ethanol concentration was substantially decreased in previously opened tubes. In the present study, ethanol concentrations decreased rapidly with time in bloodstains 
(A)

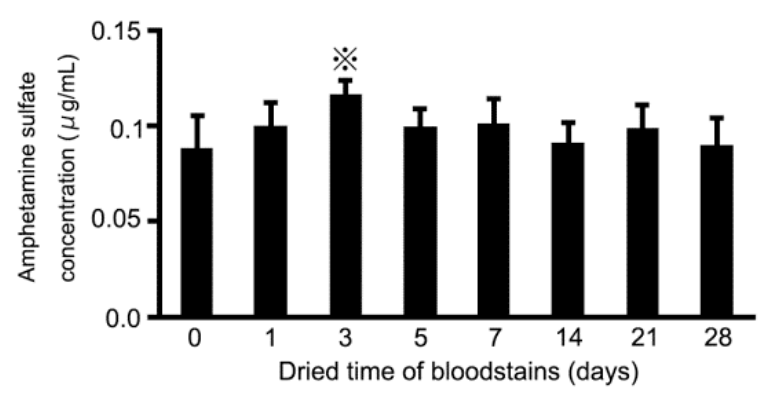

(B)

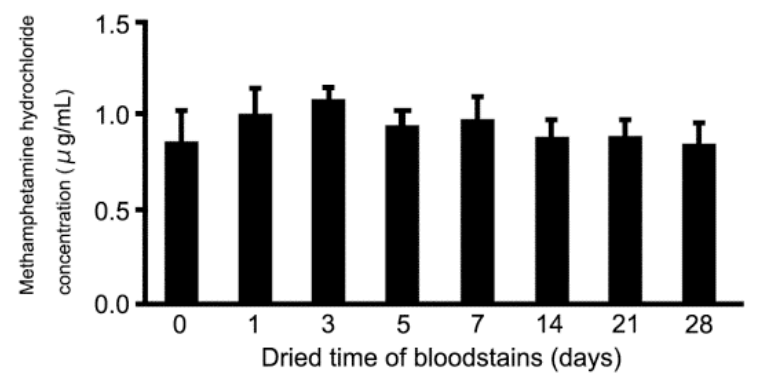

(C)

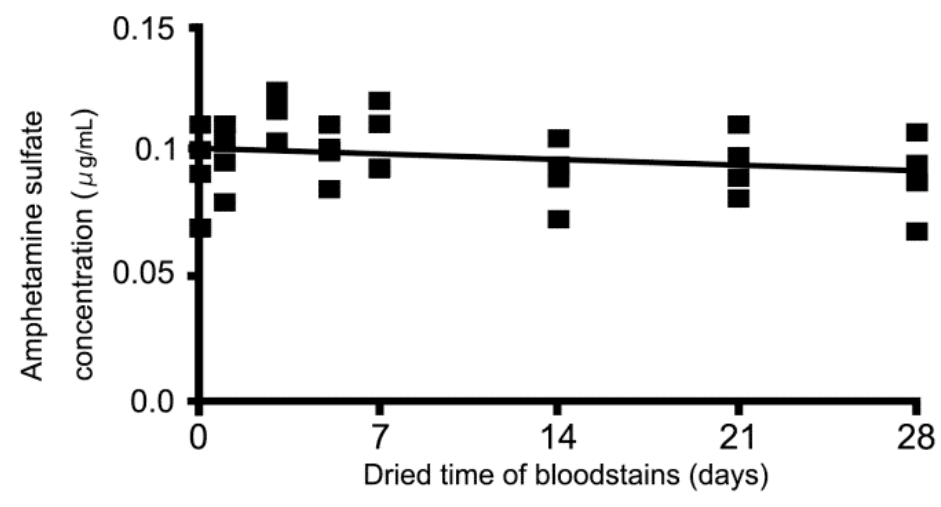

(D)

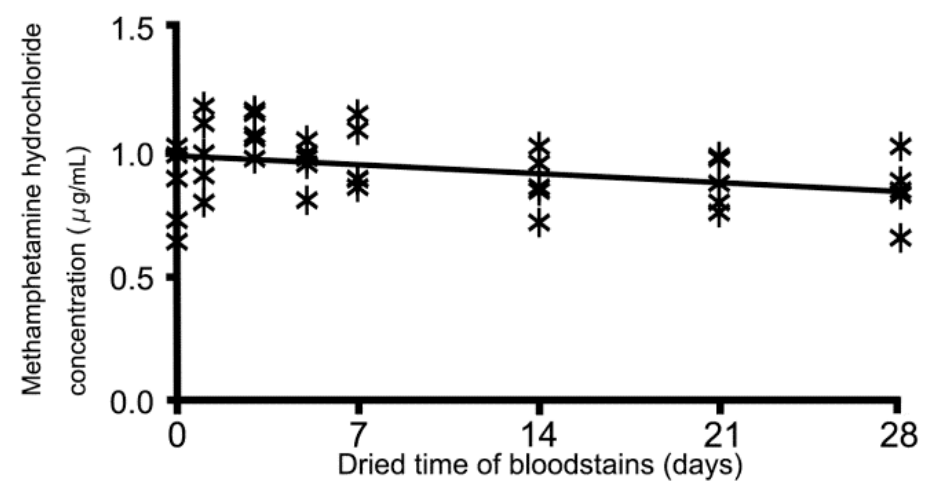

Fig. (3). Amphetamine sulfate (A, C) and methamphetamine hydrochloride (B, D) concentrations in dried bloodstains as assessed using gas chromatography-mass spectroscopy at 0-28 days after introduction into the incubation chamber. Values are expressed as mean \pm standard deviation ( $\mathrm{n}=5$ at each time point; ${ }^{*} p<0.05$ compared to the concentration of amphetamine sulfate or methamphetamine hydrochloride at day 0).

left on Petri dishes without a lid. This rapid decrease in the ethanol concentration is due to evaporation, because ethanol has a low boiling point $\left(78.4^{\circ} \mathrm{C}\right)$. Low humidity and high temperatures facilitate the evaporation of alcohol, resulting in a decrease in the alcohol concentration in bloodstains. Furthermore, it is unlikely that the chemical oxidation of ethanol can occur in such a short time period. Moreover, an evidence-based review suggests that the physiological range of ethanol elimination rates from blood is $10-35 \mathrm{mg} \cdot 100$ $\mathrm{mL}^{-1} \cdot \mathrm{h}^{-1}$ in living individuals [14]. However, in the present study, ethanol elimination rates from the dried bloodstains were approximately $100 \mathrm{mg} \cdot 100 \mathrm{~mL}^{-1} \cdot \mathrm{h}^{-1}$. Thus, prompt sampling of bloodstains at the scene of a crime or accident is required, because ethanol is eliminated much faster in bloodstains than in living persons.

There are reports on the worldwide abuse of amphetamine and methamphetamine [7,8]. The use of these stimulants can lead to confusion and exacerbates the risk of dangerous activities or probable homicidal tendencies. Moreover, the use of these illicit drugs leads to traffic accidents that require hospitalization $[7,11,15]$. Several 
studies have reported the stability of these drugs [16, 17], which is of great importance to toxicologists. Logistical limitations often lead to variation in the time intervals between sampling and analysis. In the present study, there were no noticeable changes in the concentrations of amphetamine or methamphetamine throughout the 28-day study period. Zaitsu et al. [17] reported that amphetamine and methamphetamine concentrations in urine are stable for over 150 days when stored at $25^{\circ} \mathrm{C}$ without any preservative measures. Furthermore, they reported that although the concentrations of these illicit drugs may decrease in urine due to bacterial contamination, such a decrease could be avoided by using aseptic collection kits or by adding $\mathrm{NaN}_{3}$. In the present study, all materials were used under aseptic conditions; hence, methamphetamine and amphetamine concentrations in the bloodstain samples did not decrease during the 28-day study period. Methamphetamine is known to be metabolized to amphetamine. However, we did not observe any variation in the concentration of amphetamine or methamphetamine. Therefore, it is unlikely that methamphetamine was converted to amphetamine in the bloodstains.

\section{CONCLUSIONS}

On the basis of the results of this study, we propose the following: (1) the age of bloodstains can be estimated by measuring the concentration of miRNA and analyzing the concentrations of substances contained in the bloodstains; (2) samples of bloodstains containing alcohol should be collected as quickly as possible at the scene of a crime or accident, because alcohol concentrations decrease rapidly owing to evaporation; and (3) amphetamine and methamphetamine concentrations in bloodstains may be the same as those in the blood at the time of injury. However, it should be noted that the state of a bloodstain at a crime or accident scene is affected by various environmental conditions (e.g., temperature, humidity, wind, sunlight, and pollution), the amount of bleeding, and the source of bleeding. Therefore, further studies examining the effects of such conditions will help to increase the efficiency of bloodstain analyses in criminal and traffic accident investigations.

\section{CONFLICT OF INTEREST}

The authors confirm that this article content has no conflict of interest.

\section{ACKNOWLEDGEMENTS}

The authors are extremely grateful to their colleagues for their contributions.

\section{REFERENCES}

[1] Buck, U.; Kneubuehl, B.; Näther, S.; Albertini, N.; Schmidt, L.; Thali, M.3D bloodstain pattern analysis: ballistic reconstruction of the trajectories of blood drops and determination of the centres of origin of thebloodstains. Forensic Sci. Int., 2011, 206, 22-28.

[2] Bremmer, R.H.; de Bruin, K.G.; van Gemert, M.J.; van Leeuwen, T.G.; Aalders, M.C. Forensic quest for age determination of bloodstains. Forensic Sci. Int., 2012, 216, 1-11.

[3] Anderson, S.E.; Hobbs, G.R.; Bishop, C.P. Multivariate analysis for estimating the age of a bloodstain. J. Forensic Sci., 2011, 56, 186-193.

[4] Hanson, E.K.; Lubenow, H.; Ballantyne, J. Identification of forensically relevant body fluids using a panel of differentially expressed microRNAs. Anal. Biochem., 2009, 387, 303-314.

[5] Abdel-Aty, M.A.; Abdelwahab, H.T. Exploring the relationship between alcohol and the driver characteristics in motor vehicle accidents. Accid. Anal. Prev., 2000, 32, 473-482.

[6] Gjerde, H.; Christophersen, A.S.; Normann, P.T.; Møland, J. Toxicological investigations of drivers killed in road traffic accidents in Norway during 2006-2008. Forensic Sci. Int., 2011,212, 102-109.

[7] Gustavsen, G.; Mørland, J.; Bramness, J.G. Impairment related to blood amphetamine and/or methamphetamine concentrations in suspected drugged drivers. Accid.Anal.Prev.,2006, 38, 490-495.

[8] Warren, M.W.; Kobeissy, F.H.; Liu, M.C.; Hayes, R.L.; Gold, M.S.; Wang, K.K.W. Concurrent calpain and caspase-3 mediated proteolysis of $\alpha$ II-spectrin and tau in rat brain after methamphetamine exposure: A similar profile to traumatic brain injury. Life Sci., 2005, 78, 301-309.

[9] Courts, C.; Madea, B. Micro-RNA - A potential for forensic science?Forensic Sci. Int., 2010, 203, 106-111.

[10] Patnaik, S.K.; Mallick, R.; Yendamuri, S. Detection of microRNA in dried serum blots. Anal.Biochem.,2010, 407, 147-149

[11] Appenzeller, B.M.; Schneider, S.; Yegles, M.; Maul, A.; Wenning, R. Drug and chronic alcohol abuse in drivers. Forensic Sci. Int., 2005, 155, 83-90.

[12] Mercer, S.L.; Sleet, D.A.; Elder, R.W.; Cole, K.H.; Shults, R.A.; Nichols, JL. Translating evidence into policy: Lessons learned from the case of lowering the legal blood alcohol limit for drivers. Ann Epidemiol., 2010, 20, 412-420.

[13] Shan, X.; Tiscione, N.B.; Alford, I.; Yeatman, D.T. A study of blood alcohol stability in forensic antemortem blood samples.Forensic Sci. Int., 2011, 211, 47-50.

[14] Jones, A.W. Evidence-based survey of the elimination rates of ethanol from blood with applications in forensic casework. Forensic Sci. Int., 2010, 200, 1-20.

[15] Movig, K.L.; Mathijssen, M.P.; Nagel, P.H.; van Egmond, T.; de Gier, J.J.; Leufkens, H.G.; Egberts, A.C. Psychoactive substance use and the risk of motor vehicle accidents. Accid.Anal.Prev., 2004, 36, 631-636.

[16] Clauwaert, K.M.; Van Bocxlaer, J.F.; De Leenheer, A.P. Stability study of the designer drugs "MDA, MDMA and MDEA" in water, serum, whole blood, and urine under various storage temperatures. Forensic Sci. Int., 2001, 124, 36-42.

[17] Zaitsu, K.; Miki, A.; Katagi, M.; Tsuchihashi, H. Long-term stability of various drugs and metabolites in urine, and preventive measures against their decomposition with special attention to filtration sterilization. Forensic Sci. Int., 2008, 174, 189-196. 